\title{
CENTERS OF PATH ALGEBRAS, COHN AND LEAVITT PATH ALGEBRAS
}

\author{
MARÍA G. CORRALES GARCÍA, DOLORES MARTÍN BARQUERO, CÁNDIDO MARTÍN \\ GONZÁLEZ, MERCEDES SILES MOLINA, AND JOSÉ F. SOLANILLA HERNÁNDEZ
}

\begin{abstract}
This paper is devoted to the study of the center of several types of path algebras associated to a graph $E$ over a field $K$. In a first step we consider the path algebra $K E$ and prove that if the number of vertices is infinite then the center is zero; otherwise, it is $K$, except when the graph $E$ is a cycle in which case the center is $K[x]$, the polynomial algebra in one indeterminate. Then we compute the centers of prime Cohn and Leavitt path algebras. A lower and an upper bound for the center of a Leavitt path algebra are given by introducing the graded Baer radical for graded algebras.
\end{abstract}

\section{InTRODUCTION}

The notion of center plays an essential role in algebra since its very beginning. It appears, for instance, directly related to the zero dimensional cohomology groups of algebras, immediately followed by the 1-dimensional cohomology groups, that is, derivations. On the other hand, derivations and center are related by the formula $A^{-} / Z(A) \cong \operatorname{Innder}(A)$, where $A^{-}$stands for the Lie algebra (antisimetrization) associated to the associative algebra $A$ and $\operatorname{Innder}(A)$ is the Lie algebra of inner derivations of $A$. This means that the center can be considered as the first step in the study of derivations. And motivated in part by the interest on automorphisms and derivations of path algebras, the study of the center, far from being trivial, is boarded in this work. A first attempt in the study of the center of a Leavitt path algebra was achieved by Aranda and Crow in [5], where they study the center of Leavitt path algebras and get a full description in several settings, for instance, when the Leavitt path algebra is simple.

Other interesting related problem motivating the study of the center is that of the simplicity of Lie algebras associated to Leavitt path algebras. This idea appears in the paper 4, where the authors find conditions which assures the simplicity of the Lie algebra $\left[M_{m}\left(L_{K}(n)\right)^{-}, M_{m}\left(L_{K}(n)\right)^{-}\right]$, for $L_{K}(n)$ the Leavitt algebra of type $(1, n)$, equivalently, the Leavitt path algebra of the $n$-petal rose.

2000 Mathematics Subject Classification. Primary 16D70.

Key words and phrases. Path algebra, Cohn path algebra, Leavitt path algebra, center. 
In spite of this apparently "external" arguments motivating our interest on the center of path algebras we have being also inspired by the general philosophy of finding the characterization of algebraic properties in terms of properties of the underlying graph. Since the begining of the study of Leavitt path algebras, nine years ago, many algebraic properties have being stated at a simple glance of the graph (for example simplicity, primeness, primitivity, existence of a nonzero socle and many others). In this spirit, we wanted to describe the center of a path algebra / Cohn path algebra / Leavitt path algebra only by looking at its graph.

Our initial interest was also the study of the center of graph algebras directly related to Leavitt path algebras, concretely, path algebras and Cohn path algebras. This work turned out to be illuminating for the task of the description of the center of a Leavitt path algebra.

The paper is organized as follows. We start Section 2 with some preliminaries and study the center of the path $K$-algebra $K E$ associated to a connected graph $E$, for $K$ a field; the results in this section allow us to determine the centers of prime Cohn and Leavitt path algebras in Section 3. To this task, first we reduce the study to the finite case because we obtain that if a Cohn or Leavitt path algebra has nonzero center then the number of vertices of the underlying graph must be finite. In the following step we center our attention in the 0 component of the center, which turns out to be finite dimensional, as any element it contains is proved to be symmetric. Combining this with Theorem 3 we show that if $C_{K}(E)$ is prime, it must be the Cohn path algebra associated to the $m$-petals rose graph and its center is $K$ (Subsection 3.3). In Subsection 3.4 we study the center of prime Leavitt path algebras. Here the situation is slightly more complex and we get that the center is $K$ when every cycle in the graph has an exit and the Laurent polynomial algebra $K\left[x, x^{-1}\right]$ in case there is a (necessarily unique) cycle without exits. In Section 4 we introduce a graded version of the Baer radical of a graded algebra; it turns out to be zero for any Leavitt path algebra. This result allows to prove that any Leavitt path algebra $L_{K}(E)$ is the subdirect product of a family of prime Leavitt path algebras and to conclude that the center of $L_{K}(E)$ is a subalgebra of a product of centers of prime Leavitt path algebras. We also find upper and lower bounds for the center of $L_{K}(E)$.

\section{Preliminaries and the Center of the path algebra $K E$}

We shall consider always algebras over a base field $K$. Let us fix some notation and terminology on graphs and algebras. A (directed) graph $E=\left(E^{0}, E^{1}, r, s\right)$ consists of two sets $E^{0}$ and $E^{1}$ together with maps $r, s: E^{1} \rightarrow E^{0}$. The elements of $E^{0}$ are called vertices and the elements of $E^{1}$ edges. For $e \in E^{1}$, the vertices $s(e)$ and $r(e)$ are called the source and range of $e$, respectively, and $e$ is said to be an edge from $s(e)$ to $r(e)$. If $s^{-1}(v)$ is a finite set for every $v \in E^{0}$, then the graph is called row-finite. A vertex which emits no edges is called a sink; the vertex will be called a source if it does not receive edges. A vertex $v$ is called an infinite emitter if $s^{-1}(v)$ is an infinite set, and a 
regular vertex otherwise. Also we shall use the notation $\operatorname{Path}(E)$ for the set of all paths of $E$ including the vertices as trivial paths. For a path $\lambda=e_{1} \cdots e_{n} \in \operatorname{Path}(E)$ we will call the length of $\lambda$ to the number $n$ of edges appearing in $\lambda$ and will denote it by $l(\lambda)$. Vertices are then paths of length 0 . In this case, $s(\lambda)=s\left(e_{1}\right)$ and $r(\lambda)=r\left(e_{n}\right)$ are the source and range of $\lambda$. If $\mu$ is a path in $E$, and if $v=s(\mu)=r(\mu)$, then $\mu$ is called a closed path based at $v$. If $s(\mu)=r(\mu)$ and $s\left(e_{i}\right) \neq s\left(e_{j}\right)$ for every $i \neq j$, then $\mu$ is called a cycle. An edge $e$ is an exit for a path $\mu=e_{1} \ldots e_{n}$ if there exists $i \in\{1, \ldots, n\}$ such that $s(e)=s\left(e_{i}\right)$ and $e \neq e_{i}$. Given paths $\alpha, \beta$, we say $\alpha \leq \beta$ if $\beta=\alpha \gamma$ for some path $\gamma$.

If $E_{1}=\left(E_{1}^{0}, E_{1}^{1}, s_{1}, r_{1}\right)$ and $E_{2}=\left(E_{2}^{0}, E_{2}^{1}, s_{2}, r_{2}\right)$ are graphs such that $E_{2}^{0}=E_{1}^{0}$, $E_{2}^{1}=E_{1}^{1}, s_{2}=r_{1}$ and $r_{2}=s_{1}$, then we will say that $E_{2}$ is the opposite graph of $E_{1}$. We shall use the notation $E_{2}=E_{1}^{\circ}$. It is easy to see that $K E^{\circ} \cong(K E)^{\text {op }}$, the opposite algebra of $K E$ with multiplication $x \cdot y=y x$. As a consequence there is a general principle, which we will call duality, stating that if $P$ is a property that holds for the path algebra of any graph, then a "dual property" also holds in the path algebra of any graph. If $E$ is a graph and $u, v$ two vertices, we shall say that $u$ and $v$ are connected (denoted by $u \sim v$ ) if they are connected in the underlying undirected graph of $E$ or, in other words, if there is a (finite) sequence $u=u_{1}, u_{2}, \ldots, u_{n}=v$ such that for any $i$ there is a path $\pi_{i}$ such that $s\left(\pi_{i}\right)=u_{i}, r\left(\pi_{i}\right)=u_{i+1}$ or $s\left(\pi_{i}\right)=u_{i+1}, r\left(\pi_{i}\right)=u_{i}$. We shall adopt the convention that any vertex is connected to itself by a trivial path. So, one can see immediately that connectedness is an equivalence relation whose equivalence classes will be called connected components of $E^{0}$.

If we write $E^{0}=\left\{u_{i}\right\}_{i}$ and consider the Peirce decomposition of the path algebra $A=K E$ given by $A=\oplus A_{u v}$, where $A_{u v}:=u A v$, then $A_{u v}=0$ when $u$ and $v$ are not connected. So if $E^{0}=\cup_{\alpha} E_{\alpha}^{0}$, where the $E_{\alpha}^{0}$ are the different connected components of the set of vertices, for every $\alpha$ we can define the ideals

$$
A_{\alpha}=\bigoplus_{u, v \in E_{\alpha}^{0}} A_{u v}
$$

and we have $A=\oplus_{\alpha} A_{\alpha}$. In this case it is trivial to check that $A_{\alpha} A_{\beta}=0$ when $\alpha \neq \beta$ and $Z(A)=\oplus_{\alpha} Z\left(A_{\alpha}\right)$, where $Z(\cdot)$ denotes the center of the corresponding algebra. This observation means that we can restrict our attention to those algebras $K E$ whose graph $E$ is connected.

In the mathematical field of graph theory, the distance between two vertices in an undirected graph is the number of edges in a shortest path connecting them. This is also known as the geodesic distance because it is the length of the graph geodesic between those two vertices. If there is no path connecting the two vertices, that is to say, if they belong to different connected components, then conventionally the distance is defined as infinite. The vertex set (of an undirected graph) and the distance function form a metric space if and only if the graph is connected. If $E$ is a directed graph and $u, v \in E^{0}$ are in the same connected component, we shall define the distance $d(u, v)$ as 
the geodesic distance in the underlying undirected graph associated to $E$. If $u$ and $v$ are nonconnected vertices then we shall write $d(u, v)=\infty$.

For a subset $S$ of a vector space $V$ we will denote by $\langle S\rangle$ the linear span of $S$ in $V$.

The path algebra $K E$ has a natural $\mathbb{Z}$-grading whose homogeneous components are $(K E)_{n}=0$ if $n<0,(K E)_{0}=E^{0}$ and the $n$-component, $(K E)_{n}$, for $n \geq 1$ is:

$$
\langle\{\mu \in \operatorname{Path}(E): l(\mu)=n\}\rangle .
$$

Let $X$ be a set and $T: X \rightarrow X$ a map, we define $\operatorname{Fix}(T)$ as the set

$$
\operatorname{Fix}(T):=\{x \in X: T(x)=x\} .
$$

Let $A$ be an algebra and $x \in A$, we can define the sets $\operatorname{ran}_{A}(x)=\{y \in A: x y=0\}$ and $\operatorname{lan}_{A}(x)=\{y \in A: y x=0\}$. One easy but relevant property of path algebras is the following:

Lemma 1. Let $A=K E, \mu \in \operatorname{Path}(E), u=s(\mu)$ and $v=r(\mu)$ :

(i) $\operatorname{ran}_{A}(\mu) \cap A_{v w}=0$ for all $w \in E^{0}$.

(ii) $\operatorname{lan}_{A}(\mu) \cap A_{w u}=0$ for all $w \in E^{0}$.

Proof. (i). Consider $x \in A_{v w}$ such that $\mu x=0$. Write $x=\sum_{i} k_{i} \mu_{i}$, where $k_{i} \in K$ and $\mu_{i}$ are paths with source $v$ and range $w$. We assume that the $\mu_{i}$ 's are all different. Then from $\mu x=0$ we get $\sum_{i} k_{i} \mu \mu_{i}=0$ and since all the paths in the set $\left\{\mu \mu_{i}\right\}_{i}$ are different we know that they are linearly independent. Therefore $k_{i}=0$ for all $i$ and so $x=0$. The second assertion can be proved analogously.

Observe that for an associative algebra $A$ with a system $\mathcal{E}$ of orthogonal idempotents such that

$$
A=\bigoplus_{u, v \in \mathcal{E}} A_{u v}, \quad A_{u v}=u A v
$$

the center $Z(A)$ satisfies $Z(A) \subset \bigoplus_{u \in \mathcal{E}} A_{u u}$. Thus any central element $z \in Z(A)$ admits a decomposition $z=\sum_{u \in E^{0}} z_{u}$, where $z_{u}=z u \in A_{u u}$. This decomposition will be called the Peirce decomposition of $z$ relative to $\mathcal{E}$.

Lemma 2. Let $z \in Z(K E) \backslash\{0\}$ and $u, v \in E^{0}$ such that $u \sim v$, then $z u \neq 0$ if and only if $z v \neq 0$.

Proof. If there exists $\mu \in \operatorname{Path}(E)$ such that $s(\mu)=u$ and $r(\mu)=v$, then note that

$$
z u \mu=z \mu=\mu z=\mu v z .
$$

If $z u=0$ then $\mu z v=0$, hence $z v \in \operatorname{ran}_{K E}(\mu) \cap A_{v v}=0$ by Lemma 11 (i). If $z v=0$ then $z u \mu=0$, that is, $z u \in \operatorname{lan}_{K E}(\mu) \cap A_{u u}=0$ by Lemma 1 (ii). Next we proof the general case: there is a finite sequence $u=u_{0}, u_{1}, \ldots, u_{n}=v$ such that for any $i$ there is an $f \in E^{1}$ such that $s(f)=u_{i}$ and $r(f)=u_{i+1}$ or $s(f)=u_{i+1}$ and $r(f)=u_{i}$. Then $z u_{i} \neq 0$ if and only if $z u_{i+1} \neq 0$; this proves the lemma. 
Corollary 1. If $E$ is a connected graph and $Z(K E) \neq 0$, then $\left|E^{0}\right|$ is finite.

Proof. Assume $z=\sum_{u \in E^{0}} z u \in Z(K E) \backslash\{0\}$, where only a finite number of summands is nonzero. Take a vertex $u$ such that $z u \neq 0$. For any vertex $v \in E^{0}$ we have $u \sim v$ and applying Lemma 2 we get $z v \neq 0$. Since only a finite number of summands is non zero, then $E^{0}$ must be finite.

2.1. The center of the path algebra $K E$. From now on we shall assume that $E$ has a finite number of vertices. Moreover, if $E$ is not connected it must be a finite union of finite connected graphs $E_{i}$ and $K E$ is a direct sum of the algebras $K E_{i}$. Furthermore, $Z(K E)$ is the direct sum of the centers $Z\left(K E_{i}\right)$. Hence we can focus our attention on finite connected graphs.

Lemma 3. Let $z \in Z(K E)$ with Peirce decomposition $z=\sum_{u \in E^{0}} z_{u}$. If $f \in E^{1}$ is such that $s(f)=u, r(f)=v$, then $z_{u} \in K u$ if and only if $z_{v} \in K v$.

Proof. Assume $z_{u}=k u$; since $z_{u} f=f z_{v}$ we have $k f=f z_{v}$. If $k=0$ then $z_{u}=0$ and by Lemma 2 we get $z_{v}=0$. In case $k \neq 0$ one has $\operatorname{deg}(k f)=1=\operatorname{deg}\left(f z_{v}\right)$ therefore $\operatorname{deg}\left(z_{v}\right)=0$ and $z_{v} \in K v$. Now, if $z_{v}=h v$ the same ideas lead one to the conclusion that $z_{u} \in K u$.

Proposition 1. If $u \sim v$ then $z_{u} \in K u$ if and only if $z_{v} \in K v$.

Proof. We proceed by induction on the geodesic distance $n$ between $u$ and $v$. For $n=1$ apply Lemma 3. If $n>1$ there are vertices $u=u_{1}, u_{2}, \ldots, u_{n}=v$ such that for any $i \in\{1, \ldots, n\}$ there is an arrow from $u_{i}$ to $u_{i+1}$ or from $u_{i+1}$ to $u_{i}$. So the induction hypothesis implies $z_{u_{n-1}} \in K u_{n-1}$ and then by Lemma 3, $z_{u_{n}}=z_{v} \in K v$.

As a consequence of the previous result, for a finite connected graph $E$, if $z \in Z(K E)$ and its Peirce decomposition is $z=\sum_{u \in E^{0}} z_{u}$, we have the following dichotomy:

(i) $z_{u} \in K u$ for all $u \in E^{0}$.

(ii) $z_{u} \notin K u$ for all $u \in E^{0}$.

In the first case the central element is of the form $z=\sum_{u \in E^{0}} k_{u} u$, where $k_{u} \in K$. Next we prove that all the scalars $k_{u}$ agree.

Lemma 4. Let $z \in Z(K E) \backslash\{0\}$ with Peirce decomposition of the form $z=k u+h v+$ $\sum_{w \neq u, v} z_{w}$, where $u \neq v, k, h \in K^{\times}$and $z_{w} \in A_{w w}$. If $u$ and $v$ are connected, then $k=h$.

Proof. Proceed by induction on the number $d=d(u, v)$. If $d=1$ we may assume without loss in generality that there is an $f \in E^{1}$ such that $s(f)=u$ and $r(f)=v$. Then $z f=f z$ yields $k f=h f$, hence $k=h$. Now suppose that the property holds whenever $d<n$. Consider now two vertices $u$ and $v$ such that $d(u, v)=n$. There is a vertex $w$ with $d(u, w)=1$ and $d(w, v)=n-1$. Then there exists $f \in E^{1}$ with either $s(f)=u, r(f)=w$ or $s(f)=w, r(f)=u$. In the first case, $f z=z f$ implies $f z_{w}=k f$ hence $f\left(z_{w}-k w\right)=0$ and $z_{w}-k w \in \operatorname{ran}_{K E}(f) \cap A_{w w}=0$ by Lemma 1 (i), so $z_{w}=k w$. 
Now $z=k u+h v+k w+\sum_{u^{\prime} \neq u, v, w} z_{u^{\prime}}$ and applying the induction hypothesis to $v$ and $w$ we get $k=h$. In the second case the proof is similar by using Lemma 1 (ii).

Thus for a connected finite graph $E$, the central elements are of the form (i) $z=$ $k \sum_{u \in E^{0}} u=k 1$, where $k \in K$, or (ii) $z=\sum_{u \in E^{0}} z_{u}$, where $z_{u} \notin K u$ for all $u \in E^{0}$. The elements of the form $k 1$ will be called scalars elements. From now on, we shall investigate under which conditions the path algebra $K E$ has nonscalar central elements.

Definition 1. Let $S$ denote the set of all nontrivial paths for a graph $E$. We define the map $F_{e}: S \rightarrow E^{1}$ given by $F_{e}\left(f_{1} \ldots f_{n}\right)=f_{1}$. We shall call this map the "first edge" map.

Lemma 5. Let $z$ be a nonscalar central element with Peirce decomposition $z=\sum_{v \in E^{0}} z_{v}$. If $0 \neq z_{u}=\sum_{i \in I} k_{i} \lambda_{i}$ with $k_{i} \in K^{\times}, \lambda_{i} \in \operatorname{Path}(E)$ and $f \in E^{1} \cap s^{-1}(u)$ then $f=F_{e}\left(\lambda_{i}\right)$ for each $i \in I$.

Proof. Let $v:=r(f)$ and write $z_{u}=\sum_{i \in I} k_{i} \lambda_{i}$ and $z_{v}=\sum_{j \in J} h_{j} \mu_{j}$, with $k_{i}, h_{j} \in$ $K^{\times}, \lambda_{i}, \mu_{j} \in \operatorname{Path}(E)$. Observe that $\lambda_{i}$ and $\mu_{j}$ are nontrivial paths because $z$ is nonscalar and by virtue of Proposition 1. Since $f z=z f$, weget $z_{u} f=f z_{v}$, that is,

$$
\sum k_{i} \lambda_{i} f-\sum h_{j} f \mu_{j}=0 .
$$

We claim that $\left\{\lambda_{i} f\right\} \cap\left\{f \mu_{j}\right\} \neq \emptyset$ because, otherwise, $\left\{\lambda_{i} f\right\} \cup\left\{f \mu_{j}\right\}$ would be linearly independent, so $k_{i}=0=h_{j}$ for all $i \in I, j \in J$ and therefore $z_{u}=0=z_{v}$, a contradiction. Next we prove that for every $i \in I$ there exists a unique $j \in J$ such that $\lambda_{i} f=f \mu_{j}$. Assume on the contrary that there exists $i_{0} \in I$ such that $\lambda_{i_{0}} f \notin\left\{f \mu_{j}\right\}$. Then rewrite (2) to get $k_{i_{0}} \lambda_{i_{0}} f+\sum_{i \neq i_{0}} k_{i} \lambda_{i} f-\sum h_{j} f \mu_{j}=0$. This implies $k_{i_{0}}=0$, a contradiction. Now from $\lambda_{i} f=f \mu_{j}$ we have $F_{e}\left(\lambda_{i}\right)=f$ for each $i \in I$.

Remark 1. Lemma 5 implies in particular that for a finite connected graph $E$ with nonscalar center there are no bifurcations at any $u \in E^{0}$.

Lemma 6. If $0 \neq Z(K E) \not \subset K \cdot 1$ then there are no sinks and no sources in the graph E.

Proof. By duality, it suffices to prove that there are no sinks. Assume that $v \in E^{0}$ is a sink. Let $z \in Z(K E) \backslash K \cdot 1$ be with Peirce decomposition $z=\sum_{u \in E^{0}} z_{u}$; then, by Proposition 1, each $z_{u} \notin K u$. In particular $z_{v} \notin K v$. Hence $z_{v}=\sum_{i \in I} k_{i} \mu_{i}$ with $s\left(\mu_{i}\right)=r\left(\mu_{i}\right)=v$, being $\mu_{i}$ nontrivial, which is a contradiction because $s^{-1}(v)=\emptyset$.

Proposition 2. If $0 \neq Z(K E) \not \subset K \cdot 1$ then $E$ is a cycle.

Proof. We know that $E$ is finite, connected, with no sinks and no sources (by Lemma 6) and without bifurcations (see Remark 11). Then it is easy to prove that $E$ is a cycle; by Corollary 1 we may assume $\left|E^{0}\right|=n \in \mathbb{N} \backslash\{0\}$. The proof is clear for $n=1$ so assume $n>1$. Take any vertex $u_{1}$; since it is not a sink there is a unique edge $f_{1} \in s^{-1}\left(u_{1}\right)$; 
define $u_{2}=r\left(f_{1}\right)$. If $u_{1}, \cdots, u_{i-1}$ have been defined for $i<n$ let $u_{i}=r\left(f_{i-1}\right)$, where $f_{i-1}$ is the unique edge in $s^{-1}\left(u_{i-1}\right)$. Next we proof that $u_{i} \notin\left\{u_{1}, \ldots, u_{i-1}\right\}$. Suppose on the contrary $u_{i}=u_{q}$ for $1 \leq q \leq i-1$; then $u_{i+1} \in\left\{u_{1}, \ldots, u_{i-1}\right\}$. Thus $E^{0}=\left\{u_{1}, \ldots, u_{i-1}\right\}$, which contradicts the fact that $i \leq n$. We conclude that $E^{0}=\left\{u_{1}, \ldots, u_{n}\right\}$ and as $u_{n}$ is not a sink there is only one edge $f_{n} \in s^{-1}\left(u_{n}\right)$. Now if $r\left(f_{n}\right)=u_{i}$, with $i \geq 1$, then $u_{1}$ is a source, a contradiction. Consequently $r\left(f_{n}\right)=u_{1}$ and $E$ is a cycle.

Proposition 3. If $E$ is a cycle then $Z(K E) \cong K[x]$, the polynomial algebra in the indeterminate $x$. More precisely, if $E^{0}=\left\{u_{1}, \ldots, u_{n}\right\}$ and $E^{1}=\left\{f_{1}, \ldots, f_{n}\right\}$, with $s\left(f_{i}\right)=u_{i}$ for every $i, r\left(f_{i}\right)=u_{i+1}$ for $i=1, \ldots, n-1$, and $r\left(f_{n}\right)=u_{1}$, let

$$
\begin{array}{cc}
c_{1}= & f_{1} \cdots f_{n} \\
c_{2}= & f_{2} \cdots f_{n} f_{1} \\
\vdots & \\
c_{i}= & f_{i} \cdots f_{n} f_{1} \cdots f_{i-1} .
\end{array}
$$

Then

$$
Z(K E)=\left\{\sum_{i=1}^{n} p\left(c_{i}\right): p(x) \in K[x]\right\}
$$

and there is an isomorphism from $Z(K E)$ to $K[x]$ such that $\sum_{i=1}^{n} p\left(c_{i}\right) \mapsto p$.

We collect the results and remarks above in the following

Theorem 1. If $E$ is a graph then $Z(K E)$ is the direct sum of the centers of the path algebras associated to the connected components of the graph. If $E$ is connected and has an infinite number of vertices then $Z(K E)=0$. If $E$ is connected and has a finite number of vertices then $Z(K E)=K \cdot 1$ except if $E$ is a cycle; in this case $Z(K E) \cong$ $K[x]$.

2.2. Relationship with the center of other classes of algebras. Given a graph $E$ we can define the extended graph of $E$ as the new graph $\hat{E}=\left(E^{0}, E^{1} \cup\left(E^{1}\right)^{*}, r^{\prime}, s^{\prime}\right)$, where $\left(E^{1}\right)^{*}=\left\{e^{*}: e \in E\right\}$ and the maps $r^{\prime}$ and $s^{\prime}$ are defined as $\left.r^{\prime}\right|_{E^{1}}=r,\left.s^{\prime}\right|_{E^{1}}=s$, $r^{\prime}\left(e^{*}\right)=s(e)$ and $s^{\prime}\left(e^{*}\right)=r(e)$. In this subsection we would like to establish some relationships between the center of $K \hat{E}$ and the center of certain types of algebras related to the path algebra $K \hat{E}$.

Consider the following sets of elements in $K \hat{E}$ :

$(\mathrm{CK} 1) e^{*} e^{\prime}-\delta_{e, e^{\prime}} r(e)$ for all $e, e^{\prime} \in E^{1}$. $(\mathrm{CK} 2) v-\sum_{\left\{e \in E^{1} \mid s(e)=v\right\}} e e^{*}$ for every regular vertex $v \in E^{0}$.

Then the Leavitt path $K$-algebra associated to the graph $E$, denoted $L_{K}(E)$, can be described as $L_{K}(E)=K \hat{E} / J_{1}$, where $J_{1}$ is the ideal generated by the elements in (CK1) and (CK2) (see, for example [3]). 
In the book [3] the authors define the Cohn path $K$-algebra associated to $E$, denoted by $C_{K}(E)$, as $K \hat{E} / J_{2}$, where $J_{2}$ is the ideal generated by the elements in (CK1).

In both cases the described path algebras arise as quotients of the path algebra over the extended graph module an ideal, and in order to determine their centers, we may follow a similar scheme.

So, consider an ideal $I$ of $K \hat{E}$ and the algebra $A:=K \hat{E} / I$. Then there is a short exact sequence

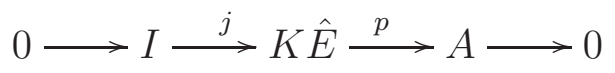

where $j$ is the inclusion and $p$ the canonical projection. Thus, for $I=J_{1}$ the algebra $A$ is isomorphic to $L_{K}(E)$ and for $I=J_{2}$ it is isomorphic to $C_{K}(E)$. Observe that $J_{i} \cap E^{1}=\emptyset$ for $i=1,2$.

Proposition 4. Let $E$ be a connected graph and $I$ an ideal of $K \hat{E}$ such that $I \cap E^{1}=\emptyset$. Define $A:=K \hat{E} / I$ and consider the short exact sequence in (3). If $E^{0}$ is finite then $\left\langle p\left(E^{0}\right)\right\rangle \cap Z(A)=K .1$; otherwise, $\left\langle p\left(E^{0}\right)\right\rangle \cap Z(A)=0$.

Proof. Take $z \in\left\langle p\left(E^{0}\right)\right\rangle \cap Z(A)$. Then $z=\sum_{k \in S} l_{k} p\left(u_{k}\right)$, with $l_{k} \in K, u_{k} \in E^{0}$ and where $S$ can be infinite but only a finite number of the $l_{k}$ 's are nonzero. Let $i \neq j$; if the geodesic distance $d\left(u_{i}, u_{j}\right)=1$ then there is an $f \in E^{1}$ with $s(f)=u_{i}$ and $r(f)=u_{j}$ (if necessary swap $i$ and $j$ ). Since $p(f) z=z p(f)$ we have $p(f) z=p(f) \sum_{k \in S} l_{k} p\left(u_{k}\right)=$ $p\left(\sum_{k \in S} l_{k} f u_{k}\right)=l_{j} p(f)$ and $z p(f)=\sum_{k \in S} l_{k} p\left(u_{k}\right) p(f)=l_{i} p(f)$. Hence $\left(l_{i}-l_{j}\right) p(f)=0$ and $\left(l_{i}-l_{j}\right) f \in I=\operatorname{Ker}(p)$. If $l_{i} \neq l_{j}$ then $f \in I \cap E^{1}=\emptyset$, a contradiction. Thus $l_{i}=l_{j}$. Suppose now that the geodesic distance $d\left(u_{i}, u_{j}\right)=n>1$; then there exists a vertex $u_{k}$ with $d\left(u_{i}, u_{k}\right)<n$ and $d\left(u_{j}, u_{k}\right)<n$. Applying a suitable induction hypothesis we have $l_{i}=l_{k}=l_{j}$. As a consequence, if $E^{0}$ is finite then $z$ is a multiple of the unit and if $E^{0}$ is infinite some scalar $l_{k}$ must be zero hence all of them are zero; therefore $z=0$.

Corollary 2. If $A$ is the Cohn path algebra or the Leavitt path algebra of a connected graph then

$$
\left\langle E^{0}\right\rangle \cap Z(A)= \begin{cases}K .1 & \text { if } E^{0} \text { is finite } \\ 0 & \text { otherwise. }\end{cases}
$$

\section{Centers of prime Cohn and Leavitt path algebras}

Once we have determined the center of $K E$ we are interested in the study of the centers of the Cohn path algebra $C_{K}(E)$ and of the Leavitt path algebra $L_{K}(E)$. As it has been said, a first step in the study of the center of a Leavitt path algebra was given in [5], where the authors determined the center of a simple Leavitt path algebra. The following natural step is to try to determine the center of prime Leavitt path algebras. 
The starting point in this section will be to prove that the existence of a nonzero center for a prime Leavitt or Cohn path algebra forces the finiteness of the number of vertices in the graph. One of the relevant tools in the theory of Cohn and of Leavitt path algebras which we shall need is the natural $\mathbb{Z}$-grading, where the vertices have degree 0 and the elements of the form $\sigma \tau^{*}$ have degree $n-m$ for $\sigma$ and $\tau$ paths of lengths $n$ and $m$ respectively. The degree of a homogeneous element $x$ in a Cohn or Leavitt path algebra will be denoted by $\operatorname{deg}(x)$.

3.1. Reduction to the finite case. The graphs $E$ in this subsection are not necessarily row-finite unless otherwise specified. Recall that for a graph $E$ a subset $H \subset E^{0}$ is said to be hereditary when for any two vertices $u, v$ such that there is a path $\mu$ with $s(\mu)=u$ and $r(\mu)=v$, if $u \in H$ then $v \in H$. A hereditary set is saturated if every regular vertex which feeds into $H$ and only into $H$ is again in $H$, that is, if $s^{-1}(v) \neq \emptyset$ is finite and $r\left(s^{-1}(v)\right) \subseteq H$ imply $v \in H$. We recall also that the ideal of the Cohn path algebra or of the Leavitt path algebra $A=C_{K}(E)$ or $L_{K}(E)$ generated by a hereditary set $H$ is easily seen to agree with the set of all linear combinations of elements of the form $\alpha \beta^{*}$, where $\alpha$ and $\beta$ are paths such that $r(\alpha) \in H$.

Lemma 7. If $A=C_{K}(E)$ or $L_{K}(E)$ and $\mu \in \operatorname{Path}(E)$ with $v=r(\mu)$, then the left multiplication operator $L_{\mu}: A \rightarrow A$ given by $a \mapsto \mu$ satisfies $\operatorname{ker}\left(L_{\mu}\right) \subset \operatorname{ran}_{A}(v)$. Moreover, $\operatorname{ker}\left(L_{\mu}\right) \cap A_{v w}=0$ for all $w \in E^{0}$.

Proof. If $L_{\mu}(a)=0$ then $\mu a=0$; therefore $\mu^{*} \mu a=0$, that is, $v a=0$ and $a \in \operatorname{ran}_{A}(v)$. If $a \in A_{v w} \cap \operatorname{ker}\left(L_{\mu}\right)$ then $a \in \operatorname{ran}_{A}(v)$; hence $v a=a\left(a \in A_{v w}\right)$ or $v a=0\left(a \in \operatorname{ran}_{A}(v)\right)$; in both cases $a=0$.

Proposition 5. Let $E$ be any graph and $A=C_{K}(E)$ or $L_{K}(E)$. Let $z \in Z(A) \backslash\{0\}$ and consider its Peirce decomposition $z=\sum_{w} z_{w}$. Fix $u, v \in E^{0}$ and assume that there is some path $\mu$ with $s(\mu)=u$ and $r(\mu)=v$; then $z_{u}=0$ implies $z_{v}=0$.

Proof. Since $z \mu=\mu z$ we get $z_{u} \mu=\mu z_{v}$. If $z_{u}=0$ we have $0=\mu z_{v}$, that is, $z_{v} \in \operatorname{ker}\left(L_{\mu}\right) \cap A_{v v}=0$.

Corollary 3. Under the conditions in the previous proposition the set

$$
H:=\left\{u \in E^{0}: z_{u}=0\right\}
$$

is hereditary.

Corollary 4. Under the conditions in the proposition above we have $A z I(H)=0$.

Proof. Take $a \in A$ and $q \in I(H)$. Then $q=\sum_{i} l_{i} \alpha_{i} \beta_{i}^{*}$, with $l_{i} \in K$ and $r\left(\alpha_{i}\right) \in H$ for each $i$. Then $a z q=a \sum_{i} l_{i} \alpha_{i} z \beta_{i}^{*}=a \sum_{i} l_{i} \alpha_{i} z r\left(\alpha_{i}\right) \beta_{i}^{*}=0$ since $r\left(\alpha_{i}\right) \in H$.

Proposition 6. If $E$ is a graph and $A=C_{K}(E)$ or $L_{K}(E)$ is prime, then $Z(A) \neq 0$ implies that $E^{0}$ is finite. 
Proof. Take a nonzero central element $z$. Since $A$ is prime and $A z, I(H)$ are ideals of $A$ whose product is zero by Corollary 4 , we conclude that either $A z=0$ or $I(H)=0$. But since $z \neq 0$ we have $A z \neq 0$. Thus $I(H)=0$, hence $H=\emptyset$ and we conclude that $z_{u} \neq 0$ for any $u$. This forces the finiteness of $E^{0}$ since the number of nonzero components in the Peirce decomposition of $z$ is necessarily finite.

Now we know that in order to have nonzero center for the prime algebras $A=C_{K}(E)$ or $L_{K}(E)$ we need finiteness of $E^{0}$.

Remark 2. It is easy to realize that for any positive integer $n$ there is a graph $E$ with $\left|E^{0}\right|=n$ such that $L_{K}(E)$ is prime and with nonzero center. Indeed, consider the graph consisting of $n$ vertices arranged in a single line. This is a simple algebra $L_{K}(E)$ whose center is $K$. However, we will prove that for a prime Cohn path algebra $C_{K}(E)$ the number of vertices in $E$ must be 1 .

Let $E$ be a row-finite graph and $A:=C_{K}(E)$. Let $u \in E^{0}$ and suppose $u$ is not a sink. Write $s^{-1}(u)=\left\{f_{i}: i=1, \ldots, n\right\}$. Then for any nontrivial path $\mu$ we have

$$
\left(u-\sum_{i} f_{i} f_{i}^{*}\right) \mu=0 \text { or, equivalently, } \mu^{*}\left(u-\sum_{i} f_{i} f_{i}^{*}\right)=0 .
$$

The element $u-\sum_{i} f_{i} f_{i}^{*}$ is an idempotent and

$$
\left(u-\sum_{i} f_{i} f_{i}^{*}\right) A\left(u-\sum_{i} f_{i} f_{i}^{*}\right)=K\left(u-\sum_{i} f_{i} f_{i}^{*}\right) .
$$

This follows by (4) and taking into account $\left(u-\sum_{i} f_{i} f_{i}^{*}\right) v=\delta_{u, v}\left(u-\sum_{i} f_{i} f_{i}^{*}\right)$. Moreover, if $v \in E^{0}$ is a sink then

$$
\left(u-\sum_{i} f_{i} f_{i}^{*}\right) A v=v A\left(u-\sum_{i} f_{i} f_{i}^{*}\right)=0
$$

and for any two different vertices $u, v \in E^{0}$ (neither of them being sinks) we have

$$
\left(u-\sum_{i} f_{i} f_{i}^{*}\right) A\left(v-\sum_{j} g_{i} g_{i}^{*}\right)=0,
$$

where $s^{-1}(u)=\left\{f_{i}\right\}$ and $s^{-1}(v)=\left\{g_{j}\right\}$.

At this point it would be convenient to recall the elementary characterization of primeness for an associative algebra: an algebra $A$ is said to be prime if and only if for any two elements $a, b \in A$ the fact $a A b=0$ implies $a=0$ or $b=0$.

At the level of Leavitt path algebras there is a purely graph-theoretic characterization of primeness. Recall that a graph $E$ satisfies Condition (MT3) if for every $v, w \in E^{0}$ there exist $u \in E^{0}$ and paths $\mu, \tau \in \operatorname{Path}(E)$ such that $s(\mu)=v, s(\tau)=w$ and 
$r(\mu)=r(\tau)=u$. In recent papers on prime Leavitt path algebras, the term "Condition (MT3)" has been replaced by the more descriptive term downward directed. It is proved in [8] that in the row-finite case, $L_{K}(E)$ is prime if and only if the graph $E$ is downward directed.

Theorem 2. Let $E$ be a row-finite graph; then the Cohn path algebra $C_{K}(E)$ is prime if and only if $\left|E^{0}\right|=1$.

Proof. Assume first that $C_{K}(E)$ is prime. Take $u, v \in E^{0}$ different. If they are not sinks then formula (7) contradicts the primeness of $A$. If one of them is a sink, then formula (6) contradicts also the primeness of the algebra. Finally, if $u$ and $v$ are sinks we have $u A v=0$ contradicting once more the primeness of the algebra. Hence $\left|E^{0}\right|=1$. Then using [3, Theorem 1.5.17] with $X=\emptyset$ the Cohn path algebra $C_{K}(E)$ is isomorphic to the Leavit path algebra $L_{K}(F)$ which is prime because $F$ is downward directed (see the graphs below).
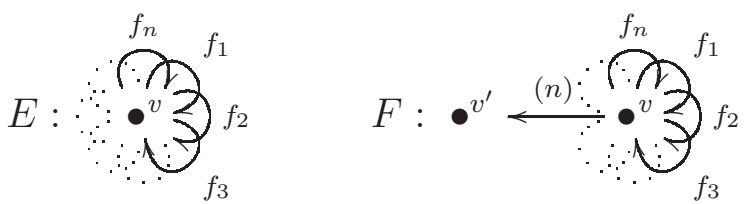

3.2. Some properties of the centers. In this subsection we shall study properties of the centers of Cohn path algebras and Leavitt path algebras that will be of interest for our purposes.

Theorem 3. Let $A$ be a $\mathbb{Z}$-graded algebra, $A=\oplus A_{n}$, with an involution $*$ such that $A_{n}^{*}=A_{-n}$ for any integer $n$. Let $Z:=Z(A)$ be its center provided with the induced $\mathbb{Z}$-grading, i.e., $Z=\oplus_{n} Z_{n}$, where $Z_{n}=Z \cap A_{n}$. Suppose that $Z$ is a domain and $Z_{0}$ a field. Then each component $Z_{n}$ is isomorphic (as a $Z_{0}$-vector space) to $Z_{0}$. Moreover either $Z=Z_{0}$ or there is an isomorphism $Z \cong Z_{0}\left[x, x^{-1}\right]$ of graded $F$-algebras.

Proof. If $Z_{n} \neq 0$ take $0 \neq x \in Z_{n}$. Then $x x^{*} \in Z_{0}$. Moreover since $Z$ is a domain $0 \neq x x^{*} \in Z_{0}$ hence $x$ is invertible. Thus any nonzero homogeneous element is invertible. Consider now the linear map $L_{x}: Z_{0} \rightarrow Z_{n}$ such that $L_{x}(a)=x a$. This is bijective since $x$ is invertible and so it is an isomorphism of $Z_{0}$ vector spaces. If there is no positive integer $n$ such that $Z_{n} \neq 0$ then $Z=Z_{0}$. Suppose on the contrary this is not true and consider the minimum positive integer $n$ such that $Z_{n} \neq 0$. Then $Z=\oplus_{i \in \mathbb{Z}} Z_{\text {in }}$ because if there exists $k \in \mathbb{Z}$ with $i n<k<(i+1) n$ and $Z_{k} \neq 0$ then $Z_{k} Z_{i n}^{*} \subset Z_{k-i n}=0$ so $Z_{k}=0$. As $Z_{n}=Z_{0} x$, then $Z_{i n}=Z_{0} x^{i}$ and $Z=\oplus_{i \in \mathbb{Z}} Z_{i n}=\oplus_{i \in \mathbb{Z}} Z_{0} x^{i}$, so $\left\{x^{i}: i \in \mathbb{Z}\right\}$ is a basis of $Z$ as a $Z_{0}$-vector space. Therefore $Z \cong Z_{0}\left[x, x^{-1}\right]$.

Recall that an algebra $A$ is graded simple if and only if $A^{2} \neq 0$ and its only graded ideals are 0 and $A$. 
Corollary 5. Let $A=L_{K}(E)$ or $A=C_{K}(E)$ be a graded simple algebra. If $Z:=$ $Z(A) \neq 0$ then $Z_{0}$ is a field and $Z=Z_{0}$ or $Z=Z_{0}\left[x, x^{-1}\right]$ (the second possibility does not occur if $A$ is simple).

Proof. Any nonzero homogeneous element in the center is invertible (because the ideal generated by this element in $A$ is graded and nonzero). In particular, $Z_{0}$ is a field. Let us prove now that $Z$ is a domain. Take $x, y \in Z$ such that $x y=0$ but $x, y \neq 0$. Write $x=\sum_{i \in \mathbb{Z}} x_{i}$, with $x_{i} \in Z \cap A_{i}$ and $y=\sum_{i \in \mathbb{Z}} y_{i}$, where $y_{i} \in Z \cap A_{i}$; consider $n:=\max \left\{i: x_{i} \neq 0\right\}$ and $m:=\max \left\{i: y_{i} \neq 0\right\}$. Since $x y=0$ we have $x_{n} y_{m}=0$, which is impossible because nonzero homogeneous elements are invertible.

Definition 2. Let $A$ be the Cohn path algebra $C_{K}(E)$ or the Leavitt path algebra $L_{K}(E)$ associated to a graph $E$. Let $z \in A_{0}$. We say that $z$ is symmetric if it is a linear combination of elements of the form $\mu \mu^{*}$, where $\mu \in \operatorname{Path}(E)$.

In what follows we will see that for $A=L_{K}(E)$ or $C_{K}(E)$ and $Z=Z(A)$, any element in the zero component of the center is symmetric, i.e., is invariant under the involution.

If $z \in Z_{0}$ and we write $z$ as a linear combination of linearly independent monomials (see [3, Proposition 1.5.6 and Corollary 1.5.11]), then every nonzero monomial in $z_{0}$ is of the form $f \mu \tau^{*} f^{*}$, where $f \in E^{1}$ or it is a scalar multiple of a vertex. To prove this statement, write $z=\sum_{i} l_{i} f \mu_{i} \tau_{i}^{*} g^{*}+r$, for $l_{i} \in K^{\times}$(where $K^{\times}=K \backslash\{0\}$ ), $f \neq g$, $f, g \in E^{1}, \mu_{i}, \tau_{i} \in \operatorname{Path}(E)$, with $\operatorname{deg}\left(\mu_{i}\right)=\operatorname{deg}\left(\tau_{i}\right)$ and $r$ stands for the remaining summands in $z$ which either do not start by $f$ or do not end by $g^{*}$ (so we have $f^{*} r g=0$ ). Then

$$
0=z f^{*} g=f^{*} z g=\sum_{i} l_{i} \mu_{i} \tau_{i}^{*} \text { implying } 0=\sum_{i} l_{i} f \mu_{i} \tau_{i}^{*} g^{*}
$$

and since the monomials $\left\{f \mu_{i} \tau_{i}^{*} g^{*}\right\}$ are linearly independent we get $l_{i}=0$, contradicting the fact that $l_{i} \in K^{\times}$. This proves that central elements of degree zero are linear combinations of vertices and monomials of the form $f \mu \tau^{*} f^{*}$, with $\mu, \tau \in \operatorname{Path}(E)$.

The set $\left\{\sigma \mu^{*}: \sigma, \mu \in \operatorname{Path}(E)\right\}$ is a basis for any Cohn path algebra (see [3, Proposition 1.5.6]). For each of these basic elements we define the real degree of $\sigma \mu^{*}$ (denoted by $\left.\partial_{\mathbb{R}}\left(\sigma \mu^{*}\right)\right)$ as the length of $\sigma$.

Definition 3. For any $\omega \in C_{K}(E)$ define $\partial_{\mathbb{R}}(\omega)=\max \left\{\partial_{\mathbb{R}}\left(\sigma_{i} \mu_{i}^{*}\right)\right\}$, where $\omega=\sum_{i \in I} l_{i} \sigma_{i} \mu_{i}^{*}$ is the expression of $\omega$ as a linear combination of the elements in the mentioned basis of the algebra.

We can also define the notion of real degree in the context of Leavitt path algebras by considering the basis given in [3, Corollary 1.5.11]. Since each element in this basis is of the form $\lambda \mu^{*}$ we can define the real degree of this element as the length $l(\lambda)$. Then the real degree of an arbitrary element can be defined as the maximum of the real degrees of the basis elements in its expression as a linear combination of the basis. 
Next we proceed to prove that any homogeneous element $z$ of degree zero in the center is symmetric.

Assume that $z=\sum_{i} l_{i} \mu f \alpha_{i} \beta_{i}^{*} g^{*} \mu^{*}+s+r$, where $l_{i} \in K, \mu, \alpha_{i}, \beta_{i} \in \operatorname{Path}(E), f, g \in E^{1}$, $f \neq g, \operatorname{deg}\left(\alpha_{i}\right)=\operatorname{deg}\left(\beta_{i}\right) \geq 0, s$ is symmetric being all its summands of real degree $\leq n$, and $r$ is a linear combination of walks whose real degrees are $>\operatorname{deg}(\mu)$ and that either do not start with $\mu f$ or do not end with $g^{*} \mu^{*}$. Observe that we can assume without loss of generality that the set of walks $\left\{\mu f \alpha_{i} \beta_{i}^{*} g^{*} \mu^{*}\right\}$ is linearly independent.

Lemma 8. We have $f^{*} \mu^{*} r \mu g=0$.

Proof. Take a summand of $r$, which we know is of the form $g_{1} \cdots g_{q} h_{q}^{*} \cdots h_{1}^{*}$, with $q>n=\operatorname{deg}(\mu)$. Then, if $\mu=t_{1} \cdots t_{n}$ we have

$$
f^{*} t_{n}^{*} \cdots t_{1}^{*} g_{1} \cdots g_{q} h_{q}^{*} \cdots h_{1}^{*} t_{1} \cdots t_{n} g \neq 0
$$

if and only if $t_{i}=g_{i}=h_{i}$ for $i=1, \ldots, n$ and $g_{n+1}=f, h_{n+1}=g$. But this implies that the summand $g_{1} \cdots g_{q} h_{q}^{*} \cdots h_{1}^{*}$ starts by $\mu f$ and ends by $g^{*} \mu^{*}$, a contradiction.

Lemma 9. We get $f^{*} \mu^{*} s \mu g=0$.

Proof. Consider a summand of $s$, say $g_{1} \cdots g_{q} g_{q}^{*} \cdots g_{1}^{*}$, with $q \leq n$. Then, in case $n>q$ and since $\mu=t_{1} \cdots t_{n}$, we have

$$
\begin{gathered}
f^{*} t_{n}^{*} \cdots t_{1}^{*} g_{1} \cdots g_{q} g_{q}^{*} \cdots g_{1}^{*} t_{1} \cdots t_{n} g=\prod_{i=1}^{q} \delta_{g_{i}, t_{i}} f^{*} t_{n}^{*} \cdots t_{q+1}^{*} t_{q+1} \cdots t_{n} g= \\
\prod_{i=1}^{q} \delta_{g_{i}, t_{i}} f^{*} g=0 .
\end{gathered}
$$

And if $n=q$ we get similarly $f^{*} t_{n}^{*} \cdots t_{1}^{*} g_{1} \cdots g_{q} g_{q}^{*} \cdots g_{1}^{*} t_{1} \cdots t_{n} g=0$.

Theorem 4. Every element of degree zero in the center of $C_{K}(E)$ or of $L_{K}(E)$ is symmetric.

Proof. Take $z \in Z_{0}$ as before. We have $0=f^{*} \mu^{*} \mu g z=f^{*} \mu^{*} z \mu g=\sum_{i} l_{i} \alpha_{i} \beta_{i}^{*}$. But the set $\left\{\alpha_{i} \beta_{i}^{*}\right\}$ is linearly independent because $\left\{\mu f \alpha_{i} \beta_{i}^{*} g^{*} \mu^{*}\right\}$ is. This implies $l_{i}=0$ and therefore $z$ is symmetric.

Definition 4. Let $A$ be an algebra with an involution $*$ and $a$ an element in $A$. Then we define the operator $T_{a}$ as the linear map $T_{a}: A \rightarrow A$ given by $T_{a}(x):=a^{*} x a$.

Note that for any $a, b \in A$ we have $T_{a} T_{b}=T_{b a}$.

Lemma 10. Let $A$ be $L_{K}(E)$ or $C_{K}(E)$ and let $f \in E^{1}$ be such that $s(f)=r(f)=u$. Consider the linear map $T_{f}: u A u \rightarrow u A u$. Then, if $\omega$ is a fixed point of $T_{f}$ of degree zero, we have $\omega=k u$ for some scalar $k$.

Proof. Assume $T_{f}(\omega)=\omega$, then $T_{f}^{n}(\omega)=\omega$ for all $n$. It is easy to see that any element $h_{1} \cdots h_{k} g_{1}^{*} \cdots g_{k}^{*}$ with $h_{i}, g_{i} \in E^{1}$ and some $h_{i} \neq f$ or some $g_{j} \neq f$ is in the kernel of $T_{f}^{m}$ for suitable $m$. Thus $\omega$ is a linear combination of elements of the form $f^{n}\left(f^{*}\right)^{m}$. But since $\operatorname{deg}(\omega)=0$ we have $n=m$. Now $T_{f}\left(f^{n}\left(f^{*}\right)^{n}\right)=f^{n-1}\left(f^{*}\right)^{n-1}$ implies that $\omega$ is a scalar multiple of $u$. 
Corollary 6. Let $E$ be a graph. If $f \in E^{1}$ is such that $s(f)=r(f)=u$, take $z \in Z_{0}=$ $Z(A)_{0}$, for $A=L_{K}(E)$ or $C_{K}(E)$. If $z=\sum z_{w}$ is the Peirce decomposition of $z$ then $z_{u} \in K u$.

Proof. Since $z f=f z$ and $s(f)=r(f)=u$ we have $z_{u} f=f z_{u}$. On the other hand we have $T_{f}\left(z_{u}\right)=f^{*} z_{u} f=f^{*} f z_{u}=z_{u}$ and by the previous lemma we conclude $z_{u} \in K u$.

Lemma 11. Let $A$ be $L_{K}(E)$ or $C_{K}(E)$ and let $c=e_{1} \ldots e_{n} \in \operatorname{Path}(E)$ be a closed path based at $u$. Consider the linear map $T_{c}: u A u \rightarrow u A u$ given by $T_{c}(x):=c^{*} x c$. If $\omega$ is a fixed point of $T_{c}$ of degree zero, then $\omega \in K u$.

Proof. First we show that for any element $z=h_{1} \cdots h_{k} g_{k}^{*} \cdots g_{1}^{*}$ the following dichotomy holds: either there is an $m$ such that $T_{c}^{m}(z)=0$ or there is an $m$ such that $T_{c}^{m}(z)=u$. To prove this, consider $z=h_{1} \cdots h_{k} g_{k}^{*} \cdots g_{1}^{*}$ such that $T_{c}^{m}(z) \neq 0$ for each $m$. Then $0 \neq T_{c}(z)=e_{n}^{*} \ldots e_{1}^{*} h_{1} \cdots h_{k} g_{k}^{*} \cdots g_{1}^{*} e_{1} \ldots e_{n}$.

If $n>k$ then $h_{i}=g_{i}=e_{i}$ for all $i \in\{1, \ldots k\}$ and $T_{c}(z)=e_{n}^{*} \ldots e_{k+1}^{*} e_{k+1} \ldots e_{n}=u$. If $n \leq k, k=q n+r$, then $T_{c}^{n q+1}(z)=\left(c^{*}\right)^{n q+1} h_{1} \cdots h_{k} g_{k}^{*} \cdots g_{1}^{*} c^{n q+1}=e_{n}^{*} \cdots e_{r+1}^{*} e_{r+1} \cdots e_{n}=$ $u$.

Now, consider a degree zero element $\omega$ such that $T_{c}(\omega)=\omega$. We can write $\omega=$ $\sum_{i \in I} t_{i} \mu_{i} \tau_{i}^{*}$, with $t_{i} \in K$ and $\mu_{i}, \tau_{i} \in \operatorname{Path}(E)$ such that $l\left(\mu_{i}\right)=l\left(\tau_{i}\right)$. Then $I=A \dot{\cup} B$, where $A$ is the set of all $i \in I$ such that $\mu_{i}, \tau_{i}^{*} \in \operatorname{ker}\left(T_{c}^{m}\right)$ for some $m$ and $B=I \backslash A$. Since $I$ is finite there exists an $m_{1}$ such that $T_{c}^{m_{1}}\left(\mu_{i} \tau_{i}^{*}\right)=0$ for each $i \in A$. For the same reason and taking into account the proved dichotomy there is an $m_{2}$ such that $T_{c}^{m_{2}}\left(\mu_{i} \tau_{i}^{*}\right)=u$ for each $i \in B$. Thus, defining $m:=\max \left(m_{1}, m_{2}\right)$ we have $\omega=T_{c}^{m}(\omega)=\left(\sum_{i \in B} t_{i}\right) u \in K u$.

Corollary 7. Let $E$ be a graph and $A=L_{K}(E)$ or $C_{K}(E)$. Take $z \in Z_{0}=Z(A)_{0}$ with Peirce decomposition $z=\sum_{v \in E^{0}} z_{v}$. If there exists a closed path $c$ based at a vertex $u$, then $z_{u} \in K u$.

Proof. Since $z c=c z$ and $s(c)=r(c)=u$ we have $z_{u} c=c z_{u}$. Moreover, $T_{c}\left(z_{u}\right)=$ $c^{*} z_{u} c=c^{*} c z_{u}=z_{u}$ and by the previous lemma we conclude $z_{u} \in K u$.

Now, suppose we are in the following situation:

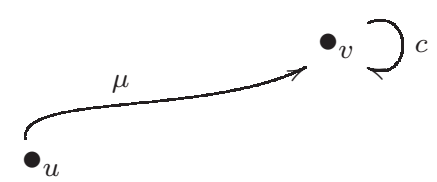

where $u, v \in E^{0}, c$ is a cycle based at $v, \mu=f_{1} \cdots f_{k}$ is a path and there is no closed path based at $s\left(f_{i}\right)$ for all $i \in\{1, \cdots, k\}$. Take $z \in Z(A)_{0}, z=\sum_{w \in E^{0}} z_{w}$; then $\mu^{*} z_{u} \mu=\mu^{*} \mu z_{v}=z_{v}$ and since $z_{v} c=c z_{v}$ we have $z_{v} \in \operatorname{Fix}\left(T_{c}\right)$, hence $z_{v}=l v$ for some $l \in K^{\times} ;$therefore

$$
\mu^{*} z_{u} \mu=l v
$$


Lemma 12. With the notation above, consider $z_{u}=k u+\sum_{i} l_{i} \sigma_{i} \sigma_{i}^{*}+\sum_{j} m_{j} \mu \gamma_{j} \gamma_{j}^{*} \mu^{*}$ with $k, l_{i}, m_{j} \in K, u \in E^{0}, \sigma_{i}, \mu, \gamma_{i} \in \operatorname{Path}(E),\{u\} \cup\left\{\sigma_{i} \sigma_{i}^{*}\right\} \cup\left\{\mu \gamma_{j} \gamma_{j}^{*} \mu^{*}\right\}$ a linear independent set and the paths $\sigma_{i}$ not of the form $\mu \gamma_{j}$. Then $\sum_{j} m_{j} \mu \gamma_{j} \gamma_{j}^{*} \mu^{*}=h_{0} \mu \mu^{*}$ for some scalar $h_{0} \in K^{\times}$.

Proof. From the expression of $z_{u}$ we get $z_{u} \mu=k \mu+\sum_{i} l_{i} \sigma_{i} \sigma_{i}^{*} \mu+\sum_{j} m_{j} \mu \gamma_{j} \gamma_{j}^{*}$. It is easy to prove that if $\sigma_{i} \sigma_{i}^{*} \mu \neq 0$ then $\sigma_{i} \sigma_{i}^{*} \mu=\mu$, so $z_{u} \mu=k \mu+\sum_{i} l_{i} \mu+\sum_{j} m_{j} \mu \gamma_{j} \gamma_{j}^{*}$ (observe that in this last sum with $l_{i}$ 's we may have less summands than in the original one). Since $\mu^{*} z_{u} \mu=k v+\sum_{i} l_{i} v+\sum_{j} m_{j} \gamma_{j} \gamma_{j}^{*}$ and by the formula (8) we have $\mu^{*} z_{u} \mu=$ $k v+\sum_{i} l_{i} v+\sum_{j} m_{j} \gamma_{j} \gamma_{j}^{*}=\lambda v$, which proves the lemma.

Proposition 7. Let $E$ be a finite and row-finite graph and $A=L_{K}(E)$ or $C_{K}(E)$. Then $\operatorname{dim}_{K}\left(Z_{0}\right)$ is finite.

Proof. We deduce from the lemma above that if there exists a path $\mu$ such that $s(\mu)=$ $u$ and $\mu$ connects to a cycle $c$ then, in the expression of $z_{u}=k u+\sum_{i} l_{i} \sigma_{i} \sigma_{i}^{*}+\lambda_{0} \mu \mu^{*}$, the cycle $c$ does not appear. We can argue as before if we consider any other cycle and path with source $u$ connecting to the cycle. Since we deal with finite graphs the set of paths which may appear in the expression of $z_{u}$ is finite and so $\operatorname{dim}_{K}\left(Z_{0}\right)$ is finite.

Lemma 13. If $A=L_{K}(E)$ or $C_{K}(E)$ is prime then $Z_{0}=Z(A)_{0}=K$.

Proof. If $K$ is algebraically closed then $Z(A)_{0}$ is a finite dimensional algebra by Proposition 7 and as $A$ is prime then $Z(A)_{0}$ is a domain so $Z(A)_{0}=K$. If $K$ is not algebraically closed, let $\Omega$ be the algebraic closure of $K$, then $Z\left(A_{\Omega}\right)=Z(A) \otimes \Omega$ and $Z\left(A_{\Omega}\right)_{0}=Z(A)_{0} \otimes \Omega$. Besides, if $A=L_{K}(E)$, then $A_{\Omega}=A \otimes_{K} \Omega=L_{K}(E) \otimes \Omega \cong L_{\Omega}(E)$ hence $A_{\Omega}$ is prime (because the primeness condition is given by a property of the graph). If $A=C_{K}(E)$ then $A_{\Omega}=A \otimes_{K} \Omega=C_{K}(E) \otimes \Omega \cong C_{\Omega}(E)$ hence $A_{\Omega}$ is also prime as before. In any case $1=\operatorname{dim}_{\Omega}\left(Z\left(A_{\Omega}\right)\right)_{0}=\operatorname{dim}_{K}\left(Z(A)_{0}\right)$ so that $Z(A)_{0}=K$.

3.3. The center of a prime Cohn path algebra. Next we will study the center of a prime Cohn path algebra $C_{K}(E)$.

Proposition 8. If $C_{K}(E)$ is prime then $E$ is the m-petals rose graph and $Z\left(C_{K}(E)\right)=$ $K$.

Proof. The primeness of $C_{K}(E)$ and the finiteness of $E$ imply that $Z\left(C_{K}(E)\right)$ is a domain. By Lemma 13 we know that $Z\left(C_{K}(E)\right)_{0}=K$ is a field. So we can apply Theorem 3 to $C_{K}(E)$ with its standard $\mathbb{Z}$-grading and involution. We conclude that $Z \cong K$ or $Z \cong K\left[x, x^{-1}\right]$. Now we must discard the second possibility. By Theorem 2 the graph $E$ must be the $m$-petals rose $R_{m}$.

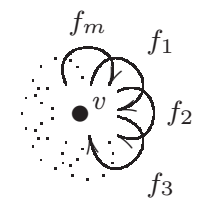


If $m=0$ then $C_{K}\left(R_{0}\right)=K v$ and there is nothing to prove. So we assume $m \geq 1$. Let $z \in Z_{n}$ with $n>0$, then $z=l_{0} \mu_{0}+l_{1} \mu_{1}\left(\gamma_{1}\right)^{*}+l_{2} \mu_{2}\left(\gamma_{2}\right)^{*}+\cdots+l_{r} \mu_{r}\left(\gamma_{r}\right)^{*}$, with $l_{i} \in K, l_{r} \in K^{\times}, \mu_{i}, \gamma_{i} \in \operatorname{Path}(E), \partial_{\mathbb{R}}\left(\mu_{0}\right)=n, \partial_{\mathbb{R}}\left(\mu_{1}\right)=n+1, \cdots, \partial_{\mathbb{R}}\left(\mu_{j}\right)=n+j$, and $\partial_{\mathbb{R}}\left(\gamma_{1}\right)=1, \cdots, \partial_{\mathbb{R}}\left(\gamma_{j}\right)=j$. We have

$$
\mu_{0}^{*} z=l_{0}+l_{1} \mu_{0}^{*} \mu_{1}\left(\gamma_{1}\right)^{*}+l_{2} \mu_{0}^{*} \mu_{2}\left(\gamma_{2}\right)^{*}+\cdots+l_{r} \mu_{0}^{*} \mu_{r}\left(\gamma_{r}\right)^{*}
$$

where $\partial_{\mathbb{R}}\left(\mu_{0}^{*} z\right) \leq r$. On the other hand

$$
z \mu_{0}^{*}=l_{0} \mu_{0} \mu_{0}^{*}+l_{1} \mu_{1}\left(\gamma_{1}\right)^{*} \mu_{0}^{*}+l_{2} \mu_{2}\left(\gamma_{2}\right)^{*} \mu_{0}^{*}+\cdots+l_{r} \mu_{r}\left(\gamma_{r}\right)^{*} \mu_{0}^{*}
$$

and now $\max \left(\partial_{\mathbb{R}}\left(z \mu_{0}^{*}\right)\right)=n+r$, whence $n+r \leq r$, which implies $n \leq 0$, a contradiction, therefore $z=0$. For $z \in Z_{-n}$, with $n>0$, we may apply the involution to get again $z=0$. Thus we conclude $Z=Z_{0}=K$.

3.4. The center of a Prime Leavitt path algebra. We begin this subsection by introducing some definitions. For a path $\mu=e_{1} \ldots e_{n}$ we denote by $\mu^{0}$ the set of vertices given by $\mu^{0}:=\left\{s\left(e_{i}\right): i=1, \ldots n\right\} \cup\left\{r\left(e_{n}\right)\right\}$.

For $X$ a nonempty subset of an algebra $A$, we denote by $I(X)$ the ideal generated in $A$ by $X$.

For a graph $E$ we will denote by $P_{c}(E)$ the set of vertices given by $P_{c}(E):=\cup \mu^{0}$, where $\mu$ ranges in the set of all cycles without exits of the graph. Recall also the so called Condition (L): we say that a graph $E$ satisfies Condition (L) if each cycle in $E$ has an exit.

The proof of the following result is contained in [2, Proposition 3.5].

Proposition 9. Let $c$ and $d$ be cycles without exits in a graph E. Then:

(i) $I\left(c^{0}\right) \cong M_{n}\left(K\left[x, x^{-1}\right]\right)$ (matrices with only a finite number of nonzero entries), where $n$ is the cardinal of the set of paths ending at the cycle $c$ and not containing all the edges of this cycle.

(ii) $I\left(c^{0}\right) I\left(d^{0}\right)=0$.

Theorem 5 (Reduction Theorem). ([, Proposition 3.1]). Let $E$ be an arbitrary graph. Then for every nonzero element $z \in L_{K}(E)$ there exist $\mu, \nu \in$ Path $(E)$ such that:

(i) $\mu^{*} z \nu=k v$ for some $k \in K \backslash\{0\}$ and $v \in E^{0}$, or

(ii) there exists a vertex $w \in P_{c}(E)$ such that $\mu^{*} z \nu$ is a nonzero polynomial $p\left(c, c^{*}\right)$, where $p\left(x, x^{-1}\right) \in K\left[x, x^{-1}\right]$.

Both cases are not mutually exclusive.

Theorem 6. Let $E$ be a graph such that $L_{K}(E)$ is a prime Leavitt path algebra, then $Z\left(L_{K}(E)\right) \neq 0$ if and only if $\left|E^{0}\right|<\infty$. In this case:

(i) $Z\left(L_{K}(E)\right) \cong K$ if and only if E satisfies Condition (L) or there exists a unique cycle without exits $c$ and infinitely many paths ending at $c$ and not containing $c$.

(ii) $Z\left(L_{K}(E)\right) \cong K\left[x, x^{-1}\right]$ if and only if $E$ contains a unique cycle without exits and there are only a finite number of paths ending at $c$ and not containing $c$. 
Proof. We observe first that if there are cycles without exits, then there is only one, since the Leavitt path algebra is prime and by condition (ii) in Proposition 9 , By Proposition 6, $Z\left(L_{K}(E)\right) \neq 0$ if and only if $\left|E^{0}\right|<\infty$. Now suppose $\left|E^{0}\right|<\infty$. Applying Theorem 3 and Lemma 13, we know that the center of $L_{K}(E)$ is isomorphic to $K$ or to $K\left[x, x^{-1}\right]$. Suppose first that $Z\left(L_{K}(E)\right) \cong K$. We will see that $E$ satisfies Condition $(\mathrm{L})$ or there exist a unique cycle without exits and infinite paths ending at $c$ and not containing $c$.

Supose that $c$ is a cycle without exits, and let $I$ be the (graded) ideal generated by $c^{0}$. By condition (i) in Proposition 9 the ideal $I$ is isomorphic to $M_{n}\left(K\left[x, x^{-1}\right]\right)$. Consider first $n \in \mathbb{N} \backslash\{0\}$. By [9, Remark 3.4] we have $Z(I)=Z\left(L_{K}(E)\right) \cap I$. Since $Z(I)$ isomorphic to $K\left[x, x^{-1}\right]$, which is infinite dimensional as a $K$-vector space and $Z\left(L_{K}(E)\right) \cong K$, we have $Z\left(L_{K}(E)\right) \cap I$ is 1-dimensional over $K$, a contradiction.

Now, suppose $Z\left(L_{K}(E)\right) \cong K\left[x, x^{-1}\right]$, and let $\varphi$ be an isomorphism from $Z\left(L_{K}(E)\right)$ into $K\left[x, x^{-1}\right]$. Take $a \in Z\left(L_{K}(E)\right)$ such that $\varphi(a)=x$; by the proof of Theorem 3 , we may suppose that $a$ is a homogeneous element of degree $d>0$. Then:

$$
\text { (†) } \quad a a^{*}=a^{*} a=1 \text {. }
$$

We claim that there exists a vertex $v$ in $E^{0}$ and $k \in K^{\times}$such that $k v a$ or $k v a^{*}$ is a power of a cycle without exits. Indeed, by the Reduction Theorem there exist paths $\mu, \nu$ such that $\mu^{*} a \nu=k v$ for some $k \in K \backslash\{0\}$ and $v \in E^{0}$, or $\mu^{*} a \nu$ is a nonzero polynomial $p\left(c, c^{*}\right)$, for $p\left(x, x^{-1}\right) \in K\left[x, x^{-1}\right]$.

In the first case, $\mu^{*} a \nu=k v$; apply that $a$ is in the center of $L_{K}(E)$ to get: $a \mu^{*} \nu=k v$. Multiply by $a^{*}$ on the left hand side of each term of this identity and apply $(\dagger)$ to get: $\mu^{*} \nu=k v a^{*}$ (note that $a^{*} \in Z\left(L_{K}(E)\right)$ ). Since the degree of $a$ is $d>0$, then $\left(\mu^{*} \nu\right)^{*}=\nu^{*} \mu=k v a$ is a path.

Because $a \in Z\left(L_{K}(E)\right)$, $k v a=\nu^{*} \mu$ is a closed path starting and ending at $v$, say $\nu^{*} \mu=e_{1} \ldots e_{n}$. We claim this closed path has no exits. Suppose on the contrary that there exists $i \in\{1, \ldots, n\}$ and $f \in E^{1}$ such that $s(f)=s\left(e_{i}\right)$ and $f \neq e_{i}$. Denote by $w$ the source of $e_{i}$. Then:

$$
\begin{gathered}
e_{i} \ldots e_{n} e_{1} \ldots e_{i-1}=\left(e_{i-1}^{*} \ldots e_{1}^{*}\right) e_{1} \ldots e_{i-1} e_{i} \ldots e_{n}\left(e_{1} \ldots e_{i-1}\right)= \\
\left(e_{i-1}^{*} \ldots e_{1}^{*}\right) k v a\left(e_{1} \ldots e_{i-1}\right)=k w a .
\end{gathered}
$$

This implies $0=f^{*} e_{i} \ldots e_{n} e_{1} \ldots e_{i-1}=f^{*}(k w a)=k f^{*} a$. Multiply by $a^{*}$ and use again $(\dagger)$. We get $0=k f^{*}$, a contradiction, and we have proved that $k v a$ is a cycle without exits.

If we are in the second case, that is, $\mu^{*} a \nu$ is a nonzero polynomial $p\left(c, c^{*}\right)$, we arrive again to the existence of a cycle without exits in $E$.

Finally we show that the number of paths ending at $c$ and not containing $c$ is finite. Suppose on the contrary that there are infinitely many paths in this situation. Since the number of vertices is finite, this means that there exists a cycle with exits $d$ connecting 
to $c$. Let $u=s(d)$. A generator system for $u L_{K}(E) u$ is $A \cup B$, for

$$
A=\left\{d^{n}\left(d^{m}\right)^{*} \mid n, m \geq 0\right\}
$$

and

$$
B=\left\{d^{n} \alpha \beta^{*}\left(d^{m}\right)^{*} \mid n, m \geq 0, s(\alpha)=u=s(\beta), d \not \leq \alpha, d \not \leq \beta, \alpha^{1} \cup \beta^{1} \not \subset d^{1}\right\} .
$$

For $n=0$ we understand $d^{n}=u$. Note that given $n, m \geq 0$ and $d^{n} \alpha \beta^{*}\left(d^{m}\right)^{*} \in B$, there exists a suitable $r \in \mathbb{N}$ such that $\left(d^{r}\right)^{*} d^{n} \alpha \beta^{*}\left(d^{m}\right)^{*} d^{r}=0$. This gives us that if we define the map $S: L_{K}(E) \rightarrow L_{K}(E)$ by $S(x)=d^{*} x d$, for every $b \in B$ there is an $n \in \mathbb{N}$ satisfying $S^{n}(b)=0$. Note that au is a fixed point for $S$. A consequence of this reasoning is that $a u \in \operatorname{span}(A)$. Write $a u=\sum_{n} k_{n} d^{n}\left(d^{m}\right)^{*}$, for $m=n-\operatorname{deg}(a)$. Then, for some $l \in \mathbb{N}$ we have $a u=S^{l}(a u)=\sum_{n} k_{n} d^{\text {deg(a) }}$. Since au commutes with every element in $u L_{K}(E) u$, the same should happen to $d^{\operatorname{deg}(a)}$, but this is not true as it does not commute with $d^{*}$, giving $a u=0$ and therefore $u=a^{*} a u=0$ (by $(\dagger)$ ), a contradiction.

\section{Consequences for the Center of A General LeavitT path algebra}

Once we know how to compute the center of a prime Leavitt path algebra, we would like to get an idea, as close as possible, on the structure of the center of a general Leavitt path algebra associated to a row-finite graph $E$. In order to achieve this target, we will give a structure theorem for Leavitt path algebras in terms of subdirect products of prime Leavitt path algebras (note that every Leavitt path algebra is a semiprime associative algebra; see, for example, [6, Proposition 1.1]). The key notion will be that of graded Baer radical whose behaviour is quite similar to that of the Baer radical (in a nongraded sense).

In this section we shall work with $\mathbb{Z}$-graded algebras and since no other grading group will be considered, the term "graded algebra" will mean $\mathbb{Z}$-graded algebra.

Let $A$ be a graded algebra, $A=\oplus_{n \in \mathbb{Z}} A_{n}$. Recall that $A$ is said to be graded semiprime when for any graded ideal $I$ of $A$ we have

$$
I^{2}=0 \text { implies } I=0 \text {. }
$$

Also $A$ is said to be graded prime when for any two graded ideals $I$ and $J$ of $A$ we have

$$
I J=0 \text { implies } I=0 \text { or } J=0 .
$$

It is easy to prove that $A$ is graded semiprime if and only if for any homogeneous element $x \in A$ we have that $x A x=0$ implies $x=0$ (which is the definition of being graded non degenerate). It is also straightforward to see that $A$ is graded prime if and only if for any two homogeneous elements $x, y \in A$ we have that $x A y=0$ implies $x=0$ or $y=0$. As a corollary we have:

$A$ is prime if and only if $A$ is graded prime, $A$ is semiprime if and only if $A$ is graded semiprime. 
The proofs of these facts can be seen in [10, Proposition II.1.4 (1)].

Recall that a graded prime ideal $I$ of $A$ is graded ideal such that $A / I$ is a graded prime algebra. In the same way we can define the notion of graded semiprime ideal.

Definition 5. Let $A$ be a graded algebra; we define the graded Baer radical of $A$ as the intersection of all graded prime ideals of $A$. We shall denote it by $\mathfrak{B}_{\mathrm{gr}}(A)$.

The following theorem is the graded version of a well-known result on the classical Baer radical. Its proof runs parallel to that of the classical theorem; we include it here for completeness.

Theorem 7. The graded Baer radical of a graded algebra $A$ is a graded semiprime ideal. In fact, it is the least graded ideal which is semiprime.

Proof. If $x \in A$ is such that $x A x \subset \mathfrak{B}_{\mathrm{gr}}(A)$ then $x A x \subset P$ for any graded prime ideal $P$. Since $P$ is semiprime we have $x \in P$ hence $x \in \mathfrak{B}_{\mathrm{gr}}(A)$. To prove that $\mathfrak{B}_{\mathrm{gr}}(A)$ is contained in any graded semiprime ideal $I$ we consider the class $\left\{P_{\alpha}\right\}_{\alpha}$ of all graded prime ideals of $A$ which contain $I$. Next we prove that $I=\cap_{\alpha} P_{\alpha}$. If there is some $x \in \cap_{\alpha} P_{\alpha}$ such that $x \notin I$ we define $x_{0}:=x$. Then $x_{0} A x_{0} \not \subset I$ (because $I$ is semiprime) and $x_{0} A x_{0} \subset \cap_{\alpha} P_{\alpha}$. Thus we can take some $x_{1} \in x_{0} A x_{0}$ such that $x_{1} \notin I, x_{1} \in P_{\alpha}$ (for any $\alpha$ ). We repeat this argument to obtain a sequence $X:=\left\{x_{0}, x_{1}, \ldots, x_{n}, x_{n+1}, \ldots\right\}$ such that $x_{n} \notin I, x_{n} \in \cap_{\alpha} P_{\alpha}$ and $x_{n+1} \in x_{n} A x_{n}$. Define the family $\mathfrak{F}$ of all graded ideals $J$ of $A$ such that $I \subset J$ and $J \cap X=\emptyset$. Since $I \in \mathfrak{F}$ we have $\mathfrak{F} \neq \emptyset$. Thus by Zorn's Lemma we have a maximal element $P \in \mathfrak{F}$. Let us prove that $P$ is a prime ideal. In order to do that take two graded ideals $J_{i}$ of $A$ such that $P \subset J_{i}$ for $i=1,2$ and $J_{1} J_{2} \subset P$. We have to prove that $P=J_{i}$ for some $i=1,2$. Suppose on the contrary $P \subsetneq J_{i}$; this implies $J_{i} \cap X \neq \emptyset$ and so some $x_{n} \in J_{1}$ and some $x_{m} \in J_{2}$. Thus, if $l \geq \max (m, n)$ we have $x_{l} \in J_{1} \cap J_{2}$ and $x_{l+1} \in J_{1} J_{2} \subset P$. But $P \in \mathfrak{F}$, which is a contradiction. So we have proved that $P$ is a prime ideal, hence it is a graded prime ideal containing $I$. Since each $x_{n} \in \cap_{\alpha} P_{\alpha} \subset P$ we have $x_{n} \in P$, which contradicts the fact that $P \cap X=\emptyset$. This proves the theorem.

Definition 6. Let $A$ be a graded algebra then we denote by $\mathfrak{s}$ the family of all graded prime ideals of $A$.

Corollary 8. Let $L_{K}(E)$ be a Leavitt path algebra. Then:

(i) $\mathfrak{B}_{g r}\left(L_{K}(E)\right)=0$.

(ii) $L_{K}(E)$ is a subdirect product of prime Leavitt path algebras.

Proof. Given that $A:=L_{K}(E)$ is graded semiprime, 0 is a graded semiprime ideal and of course it is the least graded ideal which is semiprime, so $\mathfrak{B}_{\mathrm{gr}}(A)=0$. To prove the second assertion, take into account that $\cap_{P \in \mathfrak{S}} P=\mathfrak{B}_{\mathrm{gr}}(A)=0$. Then the map 


$$
\begin{aligned}
j: A & \rightarrow \prod_{P \in \mathfrak{S}} A / P \\
a & \mapsto(a+P)_{P}
\end{aligned}
$$

is a monomorphism.

For any $Q \in \mathfrak{S}$ let $\pi_{Q}: \prod_{P} A / P \rightarrow A / Q$ be the canonical projection. Since the composition $\pi_{Q} j$ is an epimorphism then $A$ is the subdirect product of the $A / P$, which are graded prime algebras. Moreover, since each $P$ is a graded ideal then it is the ideal generated by some hereditary and saturated subset $H_{P}$ in $E^{0}$ (see [7, Lemma 2.1 and Remark 2.2]). Therefore $A / P \cong L_{K}\left(E / H_{P}\right)$, where $E / H_{P}$ denotes the quotient graph (see [7, Lemma $2.3(1)]$ ). So $A$ is the subdirect product of the Leavitt path algebras $L_{K}\left(E / H_{P}\right)$.

Take now $A$ to be the Leavitt path algebra $L_{K}(E)$. As it is known (see [7, Remark $2.2]$ ), the graded ideals and, in particular, the graded prime ideals $P \in \mathfrak{S}$ are of the form $P=I(H)$ for a unique $H \in \mathcal{H}_{E}$, where $\mathcal{H}_{E}$ stands for the hereditary and saturated subsets of $E^{0}$. The quotient algebra $A / P \cong L_{K}(E / H)$ is prime, hence the quotient graph $E / H$ is downward directed. Therefore there are three "mutually excluding possibilities" for the graph $E / H$ : either it satisfies condition (L) or it has a unique cycle without exits and a finite number of paths ending at this unique cycle and not containing it or there is a unique cycle without exits and an infinite number of paths ending at this cycle and not containing it. Thus we can classify the prime ideals $P \in \mathfrak{S}$ into two flavours:

$\mathcal{I}=\left\{I(H): H \in \mathcal{H}_{E}, E / H\right.$ is downward directed and satisfies Condition (L) $\} \cup$ $\left\{I(H): H \in \mathcal{H}_{E}, E / H\right.$ is downward directed, has a unique cycle without exits and infinitely many paths ending at this cycle and not containing it\}

$\mathcal{J}=\left\{I(H): H \in \mathcal{H}_{E}, E / H\right.$ is downward directed, has a unique cycle without exits and there is a finite number of paths ending at this cycle and not containing it\}

Theorem 8. For a row-finite graph $E$, the center of $L_{K}(E)$ is a subalgebra of $\prod_{P \in \mathcal{I}} K_{P} \times$ $\prod_{Q \in \mathcal{J}} K_{Q}\left[x, x^{-1}\right]$ containing the ideal $\bigoplus_{P \in \mathfrak{S}} Z\left(W_{P}\right)$, i.e.:

$$
\bigoplus_{P \in \mathfrak{S}} Z\left(W_{P}\right) \triangleleft Z\left(L_{K}(E)\right) \subset \prod_{P \in \mathcal{I}} K_{P} \times \prod_{Q \in \mathcal{J}} K_{Q}\left[x, x^{-1}\right]
$$

where:

(i) $K_{P}=K_{Q}=K$ for any $P \in \mathcal{I}$ and $Q \in \mathcal{J}$.

(ii) For any $P \in \mathfrak{S}$ the ideal $W_{P}$ is defined as the intersection of all the graded prime ideals others than $P$.

Proof. Let $A$ be as before the Leavitt path algebra $L_{K}(E)$. To prove that $Z(A)$ is a subalgebra of $\prod_{P \in \mathcal{I}} K_{P} \times \prod_{Q \in \mathcal{J}} K_{Q}\left[x, x^{-1}\right]$, consider $z \in Z(A)$ and let $j$ and $\pi_{Q}$ be as in the proof of Corollary [8. We want to show that $j(a)$ is in the center of $\prod_{P} A / P$. Since $\pi_{Q} j$ is an epimorphism for any $Q$, we have $\pi_{Q} j(a) \in Z(A / Q)$, so 
$a+Q \in Z(A / Q)$. Consequently, $j(a) \in Z\left(\prod_{P} A / P\right)$, which, up to isomorphism, is of the form $\prod_{P \in \mathcal{I}} K_{P} \times \prod_{Q \in \mathcal{J}} K_{Q}\left[x, x^{-1}\right]$ by Theorem 6 .

In order to prove the second assertion, we prove that the sum of the ideals $W_{P}$ is direct. Since $W_{P}=\cap_{Q \in \mathfrak{S} \backslash\{P\}} Q$ we have $W_{P} \subset Q$ for any $Q \in \mathfrak{S}, Q \neq P$. So, for any $P \in \mathfrak{S}$ we have $W_{P} \cap\left(\sum_{Q \neq P} W_{Q}\right) \subset\left(\cap_{R \in \mathfrak{S} \backslash\{P\}} R\right) \cap P=\mathfrak{B}_{\mathrm{gr}}\left(L_{K}(E)\right)=0$.

To finish the proof take into account that $Z\left(\oplus_{P} W_{P}\right)=\oplus_{P} Z\left(W_{P}\right)$ and that the center of an ideal of a semiprime algebra is contained in the center of the algebra.

The upper bound for $Z\left(L_{K}(E)\right)$ given in Theorem 8 and Theorem 6 allows to say that the building blocks for this upper bound are $K$ and $K\left[x, x^{-1}\right]$. The number of $K$ 's and $K\left[x, x^{-1}\right]$ 's appearing is completely determined by the cardinal of the sets $\mathcal{I}$ and $\mathcal{J}$. Thus it is easily computable directly from the graph $E$. On the other hand the lower bound is also algorithmically computable for a given finite graph since each $W_{P}$ is an intersection of ideals generated by hereditary and saturated subsets of the graph (hence each $W_{P}$ is also the ideal generated by some hereditary and saturated set which can be determined from the graph). So, again the ideals $W_{P}$ are Leavitt path algebras.

We have checked with several examples of concrete graphs that the center of a Leavitt path algebra may agree with the lower or with the upper bound described in Theorem 8. However our approach, the precise structure of the center remains an open question.

\section{ACKNOWLEDGMENTS}

All the authors have been partially supported by the Spanish MEC and Fondos FEDER through project MTM2010-15223, by the Junta de Andalucía and Fondos FEDER, jointly, through projects FQM-336, FQM-02467 and FQM-3737 and by the programa de becas para estudios doctorales y postdoctorales SENACYT-IFARHU, contrato no. 270-2008-407, Gobierno de Panamá. This work was done during research stays of the first and last author in the University of Málaga. Both authors would like to thank the host center for its hospitality and support.

\section{REFERENCES}

[1] G. Abrams, G. Aranda Pino, The Leavitt path algebra of a graph, J. Algebra 293 (2) (2005), 319-334.

[2] G. Abrams, G. Aranda Pino, F. Perera, M. Siles Molina, Chain conditions for Leavitt path algebras. Forum Math. 22 (2010), 95-114.

[3] G. Abrams, P. Ara, M. Siles Molina, Leavitt path algebras. A primer and handbook. Springer. To appear.

[4] G. Abrams, D. Funk-Neubauer, On the simplicity of the Lie algebras associated to Leavitt algebras. Comm. Algebra 39 (2011), 4059-4069.

[5] G. Aranda Pino, K. Crow, The Center of a Leavitt path algebra, Rev. Mat. Iber. 27 (2) (2011), 621-644.

[6] G. Aranda Pino, D. Martín Barquero, C. Martín González, M. Siles Molina, The socle of a Leavitt path algebra, J. Pure Appl. Algebra 212 (3) (2008), 500-509. 
[7] G. Aranda Pino, E. Pardo, M. Siles Molina, Exchange Leavitt path algebras and stable rank, J. Algebra 305 (2) (2006), 912-936.

[8] G. Aranda Pino, E. Pardo, M. Siles Molina, Prime spectrum and primitive Leavitt path algebras, Indiana Univ. Math. J. 58 (2) (2009), 869-890.

[9] M. Brešar, F. Perera, J. Sánchez Ortega, M. Siles Molina, Computing the maximal algebra of quotients of a Lie algebra, Forum Math. 21 (4) (2009), 601-620.

[10] C. Năstǎsescu, F. van Oystaeyen, Graded ring theory, North-Holland, Amsterdam (1982).

[11] M. TOMFORDE. Uniqueness theorems and ideal structure for Leavitt path algebras. J. Algebra 318 (2007), 270-299.

M. G. Corrales García: Centro Regional Universitario de Coclé: "Dr. Bernardo Lombardo", Universidad de Panamá. Apartado Postal 0229. Penonomé, Provincia de Coclé. Panamá.

E-mail address: mcorrales@ancon.up.ac.pa

D. Martín Barquero: Departamento de Matemática Aplicada, Escuela Técnica SuPerior de Ingenieros Industriales, Universidad de Málaga. 29071 Málaga. Spain.

E-mail address: dmartin@uma.es

C. Martín González: Departamento de Álgebra Geometría y Topología, Facultad de Ciencias, Universidad de Málaga, Campus de Teatinos s/n. 29071 Málaga. Spain.

E-mail address: candido@apncs.cie.uma.es

M. Siles Molina: Departamento de Álgebra Geometría y Topología, Facultad de Ciencias, Universidad de Málaga, Campus de Teatinos s/n. 29071 Málaga. Spain.

E-mail address: msilesm@uma.es

J. F. Solanilla Hernández: Centro Regional Universitario de Coclé: "Dr. Bernardo Lombardo", Universidad de Panamá. Apartado Postal 0229. Penonomé, Provincia de Coclé. Panamá.

E-mail address: jsolanilla@ancon.up.ac.pa 